\title{
A divergent volume for black holes calls for no "firewall"
}

\author{
Baocheng Zhang* \\ School of Mathematics and Physics, \\ China University of Geosciences, Wuhan 430074, China \\ Li You't \\ State Key Laboratory of Low Dimensional Quantum Physics, \\ Department of Physics, Tsinghua University, Beijing 100084, China and \\ Frontier Science Center for Quantum Information, Beijing, China
}

\begin{abstract}
The presumption that Hawking radiations are thermally distributed can be considered to result from their entanglement with the internal degrees of freedom for a black hole. This leads to the "firewall" paradox if unitary evolution continues into Page's time when a black hole evaporates away half of its initial entropy. However, if the interior of a black hole houses sufficient degrees of freedom to maintain entanglement with the outside at all times, unitarity can be preserved during the complete radiation process and no firewall will be required. This paper proposes a scenario that rescinds firewall by introducing the concept of volume for a black hole. Based on the operational definition by Christodoulou and Rovelli [1], we show that the volume and its associated entropy for a collapsed black hole diverges if the final evaporation stage is treated using noncommutative space. This implicates the interior of a black hole possesses adequate space to store information for a black hole of any mass, like the inside of a "magician's bag".
\end{abstract}

*Electronic address: zhangbc.zhang@yahoo.com

${ }^{\dagger}$ Electronic address: lyou@tsinghua.edu.cn 


\section{INTRODUCTION}

Since Hawking radiation was discovered [2, 3], its reconciliation with quantum theory has been continuously contested. Irrespective of what initial state a black hole starts with before collapsing, a thermal state of Hawking radiations in the end is inconsistent with unitarity required for quantum evolution. This controversy is now widely known as "the black hole

information loss paradox" [4]. Various approaches were proposed for its resolution over the past few decades (see [5-8] for recent reviews), although none has been completely successful.

With each failed attempt, the situation concerning the very existence of this paradox has become more serious. Especially after the possibility that information about infallen matter could hide into correlations between Hawking radiations and the internal states of a black hole is ruled out [9], the choice for a resolution becomes very limited. If information were not lost, it must be either taken out by the Hawking radiations or stored inside a black hole.

According to Page [10], information is leaked out through Hawking radiations, whose thermal spectrum (upon tracing out everything else), reflects their entanglement with the degrees of freedom inside a black hole. After Page's time, when half of the initial black hole entropy is evaporated, the interior of the remaining black hole would not have sufficient degrees of freedom to entangle with emitted radiations. Thus, if unitarity were preserved while information escaped out from a black hole, initial entanglement between the interior and the exterior of a black hole must be transferred to between old and young radiations. Such a presumed scenario causes the quantum field to be singular at the horizon and hence a "firewall" [11], whose crossing by a free-falling observer consequently leads to significantly different experience from the nominal case predicted by general relativity. Like "information loss paradox", this "firewall paradox" also results from the conflict between quantum mechanics and general relativity and it constitutes a renewed challenge.

If information can find a way to reside inside a black hole after Page's time, the firewall could be evaded altogether. This brings up several earlier suggestions involving remnants [12], which help to resolve the information loss paradox if they possessed infinite degrees of freedom. Quantum gravity does allow for a remnant, but its existence needs to be substantiated (more discussions can be found in the recent review [13]). Would a remnant of infinite degrees of freedom imply a satisfactory resolution for the information loss paradox? The answer, in fact, is uncertain, basically because it remains unclear how to maintain en- 
tanglement between the interior and the exterior of a black hole after Page's time, unless the black hole itself has infinite degrees of freedom in the interior regardless of its size. In particular, if a black hole with finite degrees of freedom evolves into a remnant with infinite degrees of freedom, how could this embody with unitarity?

This paper addresses the above controversy based on our recent results [14] that the volume of a noncommutative black hole is infinite. It is organized as follows. First, we will revisit the firewall paradox and discuss its intrinsic logic and the crucial elements in the second section. Then, we describe an understanding for the black hole interior that is compatible with the firewall proposal in the third section. In the forth section, we revisit the infinite volume result for a noncommutative black hole. In the fifth section, the resolution to the firewall paradox is presented based on the renewed understanding for the black hole interior with noncommutative space. Finally, we conclude in the sixth section. Throughout this paper, we use units with $G=c=\hbar=k_{B}=1$.

\section{INFORMATION LOSS PARADOX AND FIREWALL PARADOX}

Firewall at horizon was first proposed in Ref. [11] after the discovery of inconsistency with black hole complementarity [15] which states that information is not lost and no other exotic phenomena occurs within a set of postulates [11]:

P1 Unitarity of black hole evaporation: The process of formation and evaporation of a black hole, as viewed by a distant observer, can be described entirely within the context of standard quantum theory. In particular, there exists a unitary S-matrix which describes quantum theory.

P2 Validity of effective field theory: Outside the stretched horizon of a massive black hole, physics can be described to a good approximation by a set of semi-classical field equations.

P3 Existence of microscopic black hole entropy: To a distant observer, a black hole appears to be a quantum system with discrete energy levels. The dimension of the subspace of states describing a black hole of mass $\mathrm{M}$ is the exponential of the Bekenstein entropy $\mathrm{S}(\mathrm{M})$. 
P4 Equivalence principle: A freely falling observer experiences nothing out of the ordinary when crossing horizon.

First we discuss the inherent logic about these postulates. Hawking radiations are thermal, which is almost a prerequisite to investigate any problems already studied about Hawking radiations, unless the original calculation made by Hawking are found erroneous. P2 provides a natural interpretation for the spectrum due to entanglement between the outgoing Hawking mode and its interior partner mode, as a ubiquitous feature of quantum field theory. This does not lead to any conflict between the thermally distributed Hawking radiations and unitarity of quantum mechanics. But P3 states that the dimension of Hilbert space describing the radiation process of a black hole can be regarded as exp[S(M)]. Thus, when half of the initial black hole entropy is emitted away, the interior of the remaining black hole would not have sufficient degrees of freedom to entangle with the outgoing radiations. This indicates that the entanglement mechanism cannot ensure simultaneously thermal radiations and unitarity of the radiation process after the Page's time. If the sequentially emitted radiations were thermal, unitarity must be violated unless a new mechanism is found. For the present situation, unitarity or P1 requires that entanglement between the later outgoing Hawking mode $B$ and its interior partner mode $C$ is transferred to that between the early outgoing Hawking mode $A$ and $B$. Entanglement between $B$ and $C$ could ensure that Hawking radiations are thermally distributed, while the transferred entanglement $A B$ preserves the unitarity of quantum mechanics. A subtle point that is missing concerns how this transfer of entanglement takes place. Black hole complementarity tells us that such a description does not contradict with any fundamental physical principle since not a single observer can see simultaneously the two types of entanglement mentioned above.

However, Almheiri et al (AMPS) [11] found that the postulates P1, P2, P3 are inconsistent with $\mathrm{P} 4$, since they lead to the violation of strong subadditivity of the entropy,

$$
S_{A B}+S_{B C} \geqslant S_{B}+S_{A B C}
$$

The thermally distributed Hawking radiations satisfy $S_{B}>0$. Thus, this violation can be derived by making use of the expression $S_{A} \geqslant S_{B}+S_{A}$ because the first three postulates require $S_{A B} \leqslant S_{A}$ but P4 implies $S_{B C}=0$ which is equivalent to the relation $S_{A B C}=S_{A}$. In order to get rid of the contradiction, AMPS suggested that P4 should be given up, which means that free infalling observers would not feel the usual thing when crossing the 
horizon. The unusual thing called as firewall challenges our present understanding of black hole physics and leads to the breakdown of general relativity. As such it does not constitute a nice resolution to black hole information loss paradox, but erects a new paradox, called as firewall paradox, instead.

It is easily seen that the firewall paradox arises mainly from an incomplete understanding of the black hole interior [6]. Some proposals such as those involving bulk reconstruction showed no firewall, i.e. using the state-dependent operators in the black hole interior [16, 17], building a connection between entanglement and Einstein-Rosen bridge $(\mathrm{ER}=\mathrm{EPR})$ [18]. In this paper, we describe a new understanding for black hole interior with noncommutative space and investigate whether it can avoid the firewall or not.

\section{BLACK HOLE INTERIOR}

In this section we will discuss the possibility of interpreting the black hole entropy statistically by the degrees of freedom in the black hole interior. According to the definition by Christodoulou and Rovelli (CR) [1], the volume for a collapsed black hole is given by the maximal space-like hypersurface $\Sigma$ bounded by a given surface $S$. For collapsed matter described by the Eddington-Finkelstein coordinates

$$
d s^{2}=-f(r) d v^{2}+2 d v d r+r^{2} d \varphi^{2}+r^{2} \sin ^{2} \varphi d \phi^{2}
$$

with $f(r)=1-2 M / r$, the $\mathrm{CR}$ volume at late time is found to be $V_{\mathrm{CR}} \sim 3 \sqrt{3} \pi M^{2} v$, for a Schwarzschild black hole with an event horizon at $r=2 M . v=t+\int d r / f(r)=$ $t+r+2 M \ln |r-2 M|$ denotes the advanced time. The maximal hypersurface occurs at $r=$ $3 M / 2$ according to the method of auxiliary manifold [1] or maximal slicing of mathematical relativity [19]. The volume $V_{\mathrm{CR}}$ depends on the future behavior, e.g. evaporation, as it is slated to the advanced time. Furthermore, it is worth noting that the value of $V_{\mathrm{CR}}$ can be considerably larger than the observable universe for a stellar black hole.

So how many degrees of freedom can be associated with such a large interior volume? One can count the number of modes for a quantum field, e.g., a massless scalar field $\Phi$ in the spacetime with the coordinates

$$
d s^{2}=-d t_{w}^{2}+\left[-f(r) \dot{v}^{2}+2 \dot{v} \dot{r}\right] d \lambda^{2}+r^{2} d \varphi^{2}+r^{2} \sin ^{2} \varphi d \phi^{2}
$$


which results from Eq. (2) by the transformation $d v=\frac{-1}{\sqrt{-f}} d t_{w}+d \lambda$ and $d r=\sqrt{-f} d t_{w}$, and the dot on top of a variable represents partial derivative with regard to the reparameterized coordinate $\lambda$. The counting is carried out in the phase-space [20] labeled by the positions $\{\lambda, \varphi, \phi\}$ and their conjugate momenta $\left\{p_{\lambda}, p_{\varphi}, p_{\phi}\right\}$, in the space-like hypersurface at $v>>M$ and $r=3 M / 2$, and for the scalar field $\Phi$ in the volume $V_{\mathrm{CR}}$. A single quantum state corresponds statistically to a unit "cell" of phase-space volume $(2 \pi)^{3}$ and the total number of quantum states arises after integrating $d \lambda d \varphi d \phi d p_{\lambda} d p_{\varphi} d p_{\phi} /(2 \pi)^{3}$ over the complete phase space, which gives the entropy associated with $V_{\mathrm{CR}}$ as,

$$
S_{\mathrm{CR}}=\frac{\pi^{2} V_{\mathrm{CR}}}{45 \beta^{3}}
$$

for the scalar field [19, 21]. It resembles the usual relationship between entropy and volume, except that $V_{\mathrm{CR}}$ appears instead of the usual volume.

In order to estimate the entropy $S_{\mathrm{CR}}$, we calculate the parameters involved. First, a Schwarzschild black hole radiates and disappears at $v \sim M^{3}$ according to the StefanBoltzmann law, which gives a large but finite volume $V_{\mathrm{CR}} \sim M^{5}$. Then, the temperature in the black hole interior has to be worked out, which is not an easy task. But a logical assumption is to take the value of the temperature which can equates the entropy associated with the volume to the Hawking-Bekenstein entropy, $S_{\mathrm{CR}}=S_{\mathrm{H}}=\frac{A}{4}$. This caters to the original anticipation that the interior should have enough degrees of freedom to interpret statistically the black hole entropy. A straightforward calculation gives the required temperature $T=\beta^{-1}=\left(\frac{\sqrt{3} \pi^{2}}{180}\right)^{-1 / 3} / M \simeq 2.19 M^{-1}$ which is much larger than the black hole parameter $T_{\mathrm{H}}=1 / 8 \pi M$.

Irrespective whether such an interior temperature is reasonable or not, it leads to the statistical interpretation for the black hole entropy. But is such an interpretation feasible? In order for Hawking radiations to remain thermally distributed, these interior degrees of freedom must be entangled with the outgoing radiations. When the black hole evaporates half of its initial entropy away, the interior would not have enough degrees of freedom to entangle with the subsequent outgoing radiations anymore. Therefore, either the thermal spectrum has to be broken down or this entanglement is transferred to other places in order to preserve unitarity. Coincidentally, this is just the logic presented in the previous section through the postulates P1, P2 and P3. Consequently, it also conflicts with P4 and leads to the so-called firewall at the horizon. So this understanding for the black hole interior cannot 
resolve the firewall paradox.

\section{INFINITE VOLUME}

An alternative identification for the interior temperature is to take the temperature $T=$ $T_{\mathrm{H}}=1 / 8 \pi M$, and we then find Eq. (44) reduces to

$$
S_{\mathrm{CR}} \sim \frac{3 \sqrt{3}}{45 \times 8^{3}} M^{2}=\frac{3 \sqrt{3}}{90 \times 8^{4} \pi} A,
$$

with $A=16 \pi M^{2}$ the area of event horizon. This states irrevocably that entropy based on counting the modes of a scalar quantum field in $V_{\mathrm{CR}}$ is proportional to the horizon surface area that binds the interior volume [19]. Unfortunately, it remains short of providing sufficient number of modes to house all the information because the prefactor in front of $A$ is much smaller than $1 / 4$ for the Bekenstein-Hawking entropy.

The above estimate makes a critical assumption: the interior massless modes are counted at a temperature equal to that of the horizon, which is plausible if the interior and the exterior of a black hole are in thermal equilibrium. This can be confirmed by the following discussion of the black hole thermodynamics. When the volume of a black hole is taken into account, thermodynamics call for a $P d V_{\mathrm{CR}}$ like term in the free energy, whose presence would break the well established first law of black hole thermodynamics. Thanks to the entropy associated with the volume, the term $P d V_{\mathrm{CR}} \sim 10^{-5} d M$ is nicely balanced out by the $T d S_{\mathrm{CR}} \sim 10^{-5} d M$ term. Collecting all terms together, the first law of black hole thermodynamics becomes $d M=T d S-P d V_{\mathrm{CR}}$, where $S=S_{\mathrm{H}}+S_{\mathrm{CR}}$ contains both a surface $S_{\mathrm{H}}$ and a volume $S_{\mathrm{CR}}$ contribution. So what is the meaning for the pressure $P$ introduced above? It can be understood as arising from vacuum polarization [22] due to zero point fluctuations of the local energy density, which gives a quantum pressure $P=1 /\left(90 \times 8^{4} \pi^{2} M^{4}\right)$ at the horizon [23 26]. Thus, the interior temperature taken here is justified and self-consistent with black hole thermodynamics.

In this perspective, more or even infinite degrees of freedom inside a black hole are needed in order to resolve the information loss paradox. Several progresses have been made along this direction as well. First, backaction can be included when Hawking radiation is considered. The dynamic evolution for a spherically symmetric black hole due to Hawking radiation can be described by the well-known Vaidya metric [27], with which $V_{\mathrm{CR}}$ is calculated 
and the changes are however, found to be insignificant [28, 29] if the advanced time $v$ is estimated by using the Stefan-Boltzmann law. The entropy associated with $V_{\mathrm{CR}}$ including backaction is also worked out and the modification to Eq. (5) is found to be negligible as well [14].

Thus, other reasons must be found to explain the insufficient counting number (51). For instance, it could be due to the inaccurate estimate for $v$ based on the Stefan-Boltzmann law, which holds only for a black hole with a mass much greater than the Planck mass [30]. An improved treatment for the final evaporation stage can come from noncommutative space. In analogy to canonical conjugate variables in quantum mechanics, when physics enter into the Planck scale, spacetime coordinates become noncommutative, e.g., $\left[x^{\mu}, x^{\nu}\right]=i \theta \epsilon^{\mu \nu}$ where the parameter $\theta$ is of the dimension length squared. First proposed in the 40s of the last century [31], the idea of noncommutative spacetime has since been extensively applied to black hole physics (see the review [32] and references therein). Its direct application, however, is rather inconvenient. An alternative method was provided [33] which attributes the spatial noncommutative effect to a modified energy-momentum tensor, or a source while the Einstein tensor remains unchanged [34]. It is consequently found that the final evaporation stage can be well described by a remnant of mass $M_{r} \neq 0$, and the singular divergent temperature of a commutative Schwarzschild black hole is avoided.

For noncommutative space, the Eddington-Finkelstein coordinates are revised into $f(r)=$ $1-\frac{4 M}{r \sqrt{\pi}} \gamma\left(\frac{3}{2}, \frac{r^{2}}{4 \theta}\right)$, where $\gamma(\mu, z)$ denotes the lower incomplete gamma function. The modified spacetime structure dominates only near the Planck scale, but is generally negligible for a black hole of large mass. As a result, the corresponding thermodynamic parameters take their approximate (commutative) forms, including the expressions for $V_{\mathrm{CR}}$ and $S_{\mathrm{CR}}$, before approaching the Planck scale. In the final evaporation stage as $M$ approaches $M_{r}$, the temperature is approximately proportional to the mass instead of the usual inverselyproportional relationship [35]. The advanced time becomes

$$
v \simeq \frac{1}{\left(M-M_{r}\right)^{3}},
$$

in the limit of large $v$ [34]. The final evaporation stage thus needs an infinitely long time to complete, although the overall change to the black hole size will only be several $\sqrt{\theta}$. The volume associated statistical entropy formally remains the same as in Eq. (4) for 
commutative space, except for the modification to the volume itself [14]

$$
V_{\mathrm{NCR}} \sim \frac{M^{2}}{\left(M-M_{r}\right)^{3}},
$$

as $v \rightarrow \infty$. This describes a black hole with diverging volume $\left(M \rightarrow M_{r}\right)$ wrapped by a finite horizon. The associated entropy, $S_{\mathrm{NCR}}$, also diverges, although at a slower rate. A noncommutative black hole thus possesses larger information storage capacity, which indicates that its statistical interpretation for the Bekenstein-Hawking entropy must be independent of the interior [36-38]. A further refinement could include the early emission stage before Planck scale is reached. However, this change is insignificant because the estimated result from the final stage alone is already divergent.

Thus, we have presented a new scenario for the black hole interior resulting in a divergent volume. In the next section we will study whether and how the firewall can be avoided based on this result.

\section{POSSIBLE RESOLUTION TO FIREWALL PARADOX}

As stated in the second section, a crucial element for the proposal of firewall at horizon is the requirement of transferring entanglement between the outgoing Hawking radiation and its partner inside the hole to entanglement between early and later outgoing radiations. The postulate P3 indicates that the black hole interior would not have enough degrees of freedom to maintain entanglement with outgoing radiations after half of the initial entropy is emitted. However, if the black hole interior is large enough to house the required degrees of freedom during the complete radiation process, this transfer of entanglement will not be needed, which means that the firewall will be avoided.

For the situation considered in this paper, since the entropy associated volume, $S_{\mathrm{NCR}}$, is divergent, at any time of evaporation a noncommutative black hole thus always has enough degrees of freedom to entangle with outgoing Hawking radiations. This guarantees the radiations can be thermally distributed. Hence, the description about unitary evolution of black hole radiation can be changed to accommodate unitarity by assuming that information resides inside a black hole during the whole Hawking radiation process without the requirement of entanglement transfer, which hence precludes the firewall.

A brief description for the unitary process without firewall can be made accordingly by 
considering a black hole in the process of emitting radiations. Its complete state constituting

of the interior $\left|\Psi_{k}^{(i)}(\varphi)\right\rangle$ and the exterior $\left|\Psi_{k}^{(e)}\right\rangle$ (Hawking radiations) can be expressed formally as below on the right-hand-side

$$
|\varphi\rangle \rightarrow \text { evolve } \rightarrow \sum_{k} c_{k}\left|\Psi_{k}^{(i)}(\varphi)\right\rangle\left|\Psi_{k}^{(e)}\right\rangle
$$

in an entangled form, which in the end leaves Hawking radiations in a mixed state after the initial state $|\varphi\rangle$ composed of matter is collapsed into a black hole and evaporates. Of course, this scenario depends critically on whether the interior of a black hole possesses sufficient space to house information.

For the above scenario to work, the final stage of unitary evolution (8) has to be treated well. As stated in the last section, spatial noncommutativity gives a plausible description for the final stage of black hole evaporation, which provides a remnant as the ending point of evaporation. In particular, the result discussed above that a noncommutative black hole contains divergent degrees of freedom reconciles the previously discussed remnantbased resolution [13] for information loss paradox, irrespective of whether the remnants possess infinite degrees of freedom or not. A remnant possessing infinite degrees of freedom constitutes a happy ending to unitary evolution for a black hole with infinite degrees of freedom to begin with. There is no need to worry about a remnant with finite degrees of freedom because such a state cannot be reached within a finite time as long as the black hole possesses infinite degrees of freedom. In addition, if a remnant were thrown into a black hole possessing infinite degrees of freedom, no confusion or conflict with unitarity or thermodynamics will resurrect. Information loss thus do not happen for a black hole with an infinite interior. Unitary is assured and no firewall is needed.

\section{CONCLUSION}

In conclusion, the volume of a black hole is discussed for its potential and significant role in resolving black hole information loss paradox. When a black hole forms and starts to evaporate, unitary dynamics respecting Hawking's thermal radiation emissions are understood in terms of entanglement between the internal degrees of freedom and the outgoing radiations of a black hole. A plausible approach to evade firewall assumes that information is stored inside a black hole. Its validity requires the interior of a black hole to possess a 
sufficiently large volume capable of maintaining entanglement during the whole evaporation process. Our study suggests the semiclassical result for the interior volume is insufficient even for the large internal volume obtained according to the CR definition. When quantum gravity effect is taken into account using noncommutative space, the treatment for the final evaporation stage can be improved, leading to a divergent volume capable of housing infinite degrees of freedom. This presents a suggestive example to realize unitary evolution with entanglement based mechanism that calls for no firewall.

B. C. Zhang thanks Qingyu Cai for his helpful comments. This work is supported by the NSFC (grant No. 11654001, No. 11747605 and No. 91636213).

[1] M. Christodoulou and C. Rovelli, Phys. Rev. D 91 (2015) 064046.

[2] S. W. Hawking, Nature 248 (1974) 30.

[3] S. W. Hawking, Commun. Math. Phys. 43 (1975) 199.

[4] S. W. Hawking, Phys. Rev. D 14 (1976) 2460.

[5] D. Harlow, Rev. Mod. Phys. 88 (2016) 015002.

[6] J. Polchinski, arXiv: 1609.04036v1 [hep-th].

[7] W. G. Unruh and R. M. Wald, Rep. Prog. Phys. 80 (2017) 092002.

[8] A. Strominger, arXiv: 1706.07143v1 [hep-th].

[9] S. L. Braunstein and A. K. Pati, Phys. Rev. Lett. 98 (2007) 080502.

[10] D. N. Page, Phys. Rev. Lett. 71 (1993) 3743.

[11] A. Almheiri, D. Marolf, J. Polchinski, and J. Sully, J. High Energy Phys. 02 (2013) 062.

[12] Y. Aharonov, A. Casher, and S. Nussinov, Phys. Lett. B 191 (1987) 51.

[13] P. Chen, Y. C. Ong, and D-h. Yeom, Physics Reports 603 (2015) 1-45.

[14] B. Zhang and Li You, Phys. Lett. B 765 (2017) 226.

[15] L. Susskind, L. Thorlacius and J. Uglum, Phys. Rev. D 48 (1993) 3743.

[16] K. Papadodimas and S. Raju, JHEP 1310 (2013) 212.

[17] D. Marolf and J. Polchinski, JHEP 1601 (2016) 008.

[18] J. Maldacena and L. Susskind, Fortsch. Phys. 61 (2013) 781.

[19] B. Zhang, Phys. Rev. D 92 (2015) 081501(R).

[20] B. Cowan, Topics in Statistical Mechanics (Royal Holloway, Imperial College Press, London, 
UK, 2005).

[21] B. Zhang, Phys. Lett. B 773 (2017) 644.

[22] V. P. Frolov and I. D. Novikov, Black Hole Physics: Basic Concepts and New Developments (Kluwer Academic Publishers, Dordrecht, Netherlands, 1998).

[23] W. G. Unruh, Phys. Rev. D 14 (1976) 870.

[24] P. Candelas, Phys. Rev. D 21 (1980) 2185.

[25] D. N. Page, Phys. Rev. D 25 (1982) 1499.

[26] T. Elster, Phys. Lett. A 94 (1983) 205.

[27] P. Vaidya, Proc. Indian Acad. Sci. A 33 (1951) 264.

[28] Y.C. Ong, Gen. Relativ. Gravit. 47 (2015) 88.

[29] M. Christodoulou, T. D. Lorenzo, arXiv:1604.07222,

[30] S. Massar, Phys. Rev. D 52 (1995) 5857.

[31] H. S. Snyder, Phys. Rev. A 71 (1947) 38.

[32] P. Nicolini, Int. J. Mod. Phys. A 24 (2009) 1229.

[33] P. Nicolini, A. Smailagic, and E. Spallucci, Phys. Lett. B 632 (2006) 547.

[34] Y. S. Myung, Y. -W. Kim, and Y. -J. Park, JHEP 02 (2007) 012.

[35] J. M. Bardeen, B. Carter, and S. W. Hawking, Commun. Math. Phys. 31 (1973) 161.

[36] T. Jacobson, D. Marolf, and C. Rovelli, Int. J. Theor. Phys. 44 (2005) 1807.

[37] M. K. Parikh, Phys. Rev. D 73 (2006) 124021.

[38] S. Hossenfelder and L. Smolin, Phys. Rev. D 81 (2010) 064009. 